\title{
COMPORTAMIENTOS DEL CUIDADO PERCIBIDOS POR ESTUDIANTES DE ENFERMERÍA Y RECEPTORES DEL CUIDADO
}

\author{
NURSING STUDENTS AND CARE RECEIVERS’ PERCEPTIONS \\ OF CARING BEHAVIOR
}

\author{
JaCQUeline Salgado* \\ SANDRA VALENZUELA ${ }^{* *}$ \\ Katia SÁEz ${ }^{* * *}$
}

\begin{abstract}
RESUMEN
Objetivo: Determinar los comportamientos del cuidado percibido por estudiantes de último año de la carrera y la congruencia con la percepción de los mismos, por los usuarios receptores del cuidado. Material y método: Estudio descriptivo, transversal, realizado el año 2012 en estudiantes de Enfermería de Internado y receptores del cuidado de éstos, de tres instituciones de formación universitaria, comuna de Concepción, Chile. Muestra conformada por 96 internas/os y 96 receptores del cuidado. Se utilizó el Cuestionario CBI de Zane Wolf, basado en la Teoría del cuidado humano de Jean Watson, versiones estudiante y usuario. Resultados: Los estudiantes presentan edades entre 21 y 33 años, cursaron entre 8 y 6 años antes de alcanzar el $5^{\circ}$ nivel. Los estudiantes de las universidades 2 y 3 desarrollaron práctica en servicios generales, en tanto los de la universidad 1 lo hicieron en unidades de especialidad. El promedio de edad de los usuarios fue de 54,6 años. En relación a los comportamientos del cuidado brindado, referidos por internos para las dimensiones 1, 2 y 5, existió una sobreestimación de percepción de los estudiantes acerca del cuidado brindado, por sobre la percepción referida por usuarios. En la dimensión 4, “conocimiento y destreza profesional”, los estudiantes de las universidades 2 y 3 subestiman sus comportamientos de cuidado respecto a lo percibido por usuarios. Conclusión: Los usuarios estarían en condiciones de evaluar asertivamente comportamientos del cuidado humanizado en dimensiones 1, 2, 3 y 5, y no así, el conocimiento y destreza profesional de la dimensión 4.
\end{abstract}

Palabras clave: Comportamientos del cuidado, estudiantes de enfermería, cuidado de enfermería.

\begin{abstract}
Objective: To analyze nursing students and care receiver's perceptions of caring behavior. Method: Descriptive study carried out in 2012, on nursing students' internship and care receivers from three institutions of higher education located in Concepción, Chile. Data were collected from a sample of 96 interns and 96 care receivers. Caring Behaviours Inventory (CBI) developed by Zane Wolf and based on the theory of human caring proposed by Jean Watson, was used in a version for students and another of users. Results: Students aged 21 to 33 years who studied between 8 and 6 years before reaching the fifth level. Students from the university 2 and 3 performed a

\footnotetext{
*Enfermera. Docente Universidad San Sebastián. Email: jsalgado@uss.cl

${ }^{* *}$ Enfermera. Docente Facultad de Enfermería. Universidad de Concepción. Email: svalenzu@udec.cl

${ }^{* * *}$ Ingeniero Matemático. Profesora Facultad de Ciencias Físicas y Matemáticas, Universidad de Concepción, Email: ksaez@ udec.cl
} 
practice in general services while the students of university 1 did it in (health) specialist units. The average age of the users was 54.6 years old. In relation to the behaviors of the care provided, refered by interns there was an overestimation of the students' perceptions about the care provided above the perception reported by the users for dimensions 1, 2 and 5. In dimension 4, "professional knowledge and skills", the students form universities 2 and 3 underestimate their caring behaviors in relation to the users' perception. Conclusion: Users would be able to assertively evaluate behaviors of humanized care in the dimensions 1, 2, 3, and 5. But concerning dimension 4 "professional knowledge and skills", would not.

Key words: Caring behaviors, nursing students, nursing care.

Fecha recepción: 15/09/13 Fecha aceptación: 22/03/15

\section{INTRODUCCIÓN}

En Chile, la enseñanza de enfermería ha experimentado una rápida expansión en los últimos años. De 17 programas en la década de los 80 en el país, se observa ya, en el año 2006, la existencia de 65 Escuelas de enfermería, con una matrícula de 12.407 estudiantes, de las cuales el $75 \%$ fueron creadas a partir del año 2000. Actualmente se encuentran 110 programas en Chile, lo que representa un incremento de $61 \%$ en la última década. Por otra parte, la carrera de Enfermería se ubica como la segunda profesión más elegida por los postulantes que acceden a la educación superior, en vista de las oportunidades y ofertas laborales aseguradas (1).

El perfil de egreso descrito en la mayoría de estos programas determina un profesional preparado para gestionar y otorgar cuidado humanizado, holístico, centrado en el paciente, su familia y en su entorno social y cultural (2). Por otro lado, la marcada expresión del proceso médico con su enfoque de problema de salud aún persistente en nuestro medio, dificulta la configuración del proceso de cuidar desde una perspectiva focalizada en la respuesta humana a las condiciones de salud y enfermedad y en la persona desde una visión holística, en relación con su entorno (3).

Por ello, se hace imperativo impregnar a los estudiantes de Enfermería, durante el proceso académico, de cuidar con empatía, con respeto, independiente del diagnóstico y de los modelos de atención que tenga para ello. El realizar un procedimiento con altos estándares de calidad, es otra manera de cuidar con respeto, pero según los mismos usuarios el hacerlo bien no es tan importante para ellos como hacerlo con entrega y empatía (4).

Investigar sobre la percepción de conductas de cuidado en estudiantes de Enfermería podría llevar a definir las influencias que la formación universitaria y profesional tienen sobre éste, ya que la percepción de comportamientos del cuidado influye sobre el desarrollo del acto mismo de cuidado, en el sentido que repercute en la forma como las/ os enfermera/os, y a futuro los estudiantes de enfermería, actúan y actuarán dentro del acto de cuidado de enfermería y en la forma como el paciente responde o responderá al mismo (5).

La razón fundamental que motivó la realización de este estudio radica en la escasa presencia de trabajos que aborden los comportamientos del cuidado de las y los estudiantes de Enfermería, su percepción respecto a éstos, como evidencia de la congruencia entre el discurso teórico actual de la enfermería y su ejercicio en las variadas instituciones formadoras; contrastando además, con la percepción del receptor del cuidado; todo esto respaldado por instrumentos que midan los Comportamientos del Cuidado. 


\section{Marco referencial}

El espacio en que aprenden los futuros profesionales de salud coloca poco valor sobre el contenido relacionado con la humanización del cuidado, mientras el contenido técnico, relacionado exclusivamente con los aspectos biológicos del ser humano, es sobreevaluado, lo que influye durante la formación profesional, repercutiendo en las actitudes profesionales $(6,7)$.

Watson sostiene que "ante el riesgo de deshumanización en el cuidado del paciente, a causa de la gran reestructuración administrativa de la mayoría de los sistemas de cuidado de salud en el mundo, se hace necesario el rescate del aspecto humano, espiritual y transpersonal" (8).

Los estudiantes en práctica de Internado, denominados internas/os de enfermería, son los que cursan en este caso el $5^{\circ}$ año de su malla curricular de pregrado, etapa considerada como actividad indispensable para consolidar las competencias necesarias, establecer capacidades cognitivas, procedimentales $y$ actitudinales, para el futuro ejercicio profesional y desempeño del rol en sus dimensiones: asistencial, educador, gestor e investigador. En esta práctica de internado se integran a los conocimientos técnicos y científicos, a la fundamentación teórica y, principalmente, a la formación humanística y ética, aspectos esenciales para el desempeño profesional que se desarrolla a través de las relaciones humanas (8).

Entonces, basado en lo anterior, podría interpretarse que hasta la fecha las instituciones de educación superior de enfermería egresan profesionales formados integralmente, es decir, con el conjunto de conocimientos, habilidades y valores que garantizan un cuidado humanizado. Sin embargo, este supuesto encuentra detractores que afirman que esta situación de egreso no es homogénea en la región, donde persisten aún programas que se sustentan en el modelo biomédico y en el morbicentrismo (9).
Respecto a esta importante etapa de internado, es necesario destacar que en la Región del Bío-Bío se desarrolla con una modalidad anual, con dos áreas de experiencias: Hospitalaria y Comunitaria; en este periodo el interno debe incorporarse plenamente en los equipos de trabajo, desarrollando actividades que permitan cumplir objetivos y competencias, determinados en los respectivos programas orientados a facilitar la inserción en los espacios laborales de sus egresados.

Las instituciones que acogen a estos internos/as a su vez, deben cautelar el cumplimiento de los marcos ético legales establecidos por el Ministerio de Salud chileno, en cumplimiento a la Ley $\mathrm{N}^{\circ} 20.584$ de Derechos y Deberes de los Pacientes (10), donde el cuidado humanizado se hace preponderante y obligatorio; pero ¿cómo y con qué instrumento medir estos aspectos?

La revisión de las publicaciones con respecto a la identificación de los cuidados de enfermería por parte de los profesionales, nos lleva a afirmar que todavía se presentan dificultades para identificarlos en la práctica, a pesar de estar bien definidos en los modelos de cuidados y en innumerables publicaciones. Esto porque la Percepción del Cuidado aún se confunde con la Atención de Enfermería, diluyéndose en la atención que involucra preponderantemente aspectos técnicos y procedimentales. Esta confusión a su vez provoca en los estudiantes, muchas veces, una percepción ambivalente y ambigua, donde se confunde el tratamiento con el cuidado (7). Este instrumento (CBI) logra enfatizar claramente los aspectos centrales del cuidado humanizado de Enfermería, pues especifica la real composición de estos elementos del cuidado, conceptualizando en sus cinco dimensiones las áreas que componen el cuidado profesional de enfermería: deferencia respetuosa con otro, asegura la presencia humana, conectarse positivamente, conocimiento y destreza profesional y prestar atención a la experiencia de otros; evitando así la preponderancia de uno de los componentes 
sobre los otros, tanto para los cuidadores como para los usuarios.

La conceptualización elaborada por el grupo de cuidado de la Facultad de Enfermería de la Universidad Nacional de Colombia entiende la percepción del cuidado como: "Los elementos y las características del ser cuidado y del cuidar al otro con relación al entorno situacional y al escenario en que se da el acto de cuidado. Es el proceso mental mediante el cual el paciente y el profesional de enfermería identifican los comportamientos del cuidado de acuerdo con sus criterios, valores, vivencias, experiencias y expectativas" (11).

La noción del cuidado se ha constituido como el imperativo moral y principal valor regulativo de la formación de enfermeras/ os; se postula la necesidad de un cambio, que busque un isomorfismo entre la formación recibida por el estudiante y el tipo de cuidado que se le va a pedir que desarrolle como profesional (12).

Los elementos de la teoría de Watson han dado orientación a una continua evolución en la creación de variados instrumentos que intentan una aproximación cuantitativa al fenómeno del cuidar a través de la construcción de indicadores empíricos. La importancia de poder operacionalizar el objeto de estudio de la enfermería radica en que para que el conocimiento de enfermería se incremente, progrese y se difunda es importante que sea entendido y comprendido desde la formación y el ejercicio profesional.

Los instrumentos son importantes para el investigador, ya sea para la obtención de datos o para acercarse al fenómeno y extraer de ellos información; además se presenta como una herramienta útil para extraer evidencia científica que fundamente el real cumplimiento de los requerimientos educacionales y asistenciales ético-legales y de gestión establecidos por las instituciones prestadoras de servicios. Para Enfermería, el cuidado se ha convertido en el centro de la praxis, y evaluar que estos cuidados sean humanizados beneficia a los profesionales de Enfermería y a los usuarios, mejorando la calidad de éstos en los servicios de salud (13).

Para Watson, el no delimitar el campo propio de realización de enfermería y referirlo a un campo biomédico no hace posible la autonomía y la creatividad, por tanto, para cumplir la misión de brindar cuidados efectivos es indispensable cambiar esta imagen, adquirir autoconfianza y ejercer más autonomía. Para ello, el instrumento privilegiado es la investigación, el aprendizaje, la apertura a nuevas estrategias de cuidados, establecer correcciones a las teorías existentes y elaborar nuevas. En resumen, es vital desarrollar un marco de conocimientos respecto al cuidado (14).

Diversos estudios (15-19) demuestran la importancia de los comportamientos del cuidado de las enfermeras asistenciales y de las docentes, en la preparación de los estudiantes para el desarrollo de las prácticas clínicas, presentando evidencia acerca de la necesidad de obtener resultados de más de un actor del ejercicio del cuidado, para el efecto de modelado del cuidado transpersonal. El cuidado es un valor básico y un atributo en los estudiantes de Enfermería, pero el proceso educativo en algunos casos podría llegar a reducir estas conductas de cuidado. Confirman la necesidad de evaluar constantemente el proceso enseñanza-aprendizaje de los estudiantes utilizando instrumentos validados y eficientes en cada contexto. Sugieren, además, contrastar la información obtenida y detectar las áreas susceptibles de ser modificadas en beneficio de la satisfacción usuaria, triangulación de la información obtenida, estableciendo, así, fundamentos científicos sólidos para la reorientación y retroalimentación del cuidado de enfermería.

El objetivo fue determinar los comportamientos del cuidado percibido por estudiantes de último año de la carrera y la congruencia con la percepción de los mismos por los usuarios receptores del cuidado, por medio de un instrumento confiable para estos fines, presentado en dos versiones (estudiantes y usuarios), que podrían ser de utilidad para 
los procesos de acreditación de los centros docente-asistenciales.

\section{MATERIAL Y MÉTODO}

El presente estudio, de abordaje cuantitativo, de tipo descriptivo, transversal (20), fue realizado en Chile durante el segundo semestre del año 2012.

La unidad de análisis correspondió a un binomio compuesto por: Estudiante de Enfermería que cursa $5^{\circ}$ nivel de la carrera, que realiza su experiencia práctica de Internado en institución de salud y Usuario/a que recibe cuidado de estudiante de Enfermería en institución de salud en Concepción, Chile, 2012.

El Universo correspondió a todos los estudiantes de Enfermería que cursan $5^{\circ}$ nivel de la carrera de 3 universidades (una de ellas perteneciente al Consejo de Rectores, denominada universidad 2), que realizan sus experiencias prácticas de internado hospitalarias en instituciones de salud y todos los usuarios que recibieron cuidado de cada estudiante de Enfermería que estaba cursando su práctica. Se consideró un usuario receptor de cuidados de cada estudiante que reuniera los requisitos de inclusión establecidos en el estudio.

Población del estudio: Constituida por el Universo de internas/os de enfermería $(\mathrm{N}=96)$ en ejercicio de su experiencia clínica de internado segunda rotación y por cada estudiante, un usuario $(\mathrm{N}=96)$ que recibió cuidado de enfermería de éste durante el $2^{\circ}$ semestre del año académico 2012.

Criterios de inclusión: Todos los estudiantes de Enfermería que cursan $5^{\circ}$ nivel de la carrera, que realizan sus experiencias prácticas de internado en instituciones de salud, con al menos 1 mes de permanencia previa en el referido servicio clínico. Todos los usuarios que reciben cuidado de estos estudiantes de Enfermería, con al menos tres días de permanencia en el servicio clínico cumplidos al momento de su alta; en pleno uso de sus fa- cultades y que aceptaron participar voluntariamente en el estudio previo consentimiento informado. Concepción, Chile, 2012.

La recolección de los datos se realizó a través del método de encuesta para el estudiante y usuario. Se aplicaron los Cuestionarios 1 y 2 sobre antecedentes estudiante y usuario respectivamente y Cuestionario 3 y 4 Caring Behaviors Inventory (CBI) de Zane Wolf (facilitados por la autora a los investigadores) en sus dos versiones: para estudiante y usuario.

Descripción de los instrumentos recolectores de datos:

Instrumento $\mathrm{N}^{\circ}$ 1: Cuestionario elaborado por las autoras de este estudio sobre variables descriptivas personales y académicas: edad; sexo; motivos de ingreso a la carrera; universidad donde cursa estudios; lugar de experiencia práctica; duración y tiempo de experiencia práctica; formación teórica (apreciación); formación práctica (apreciación); modelo de enfermería estudiado; taxonomía utilizada en planificación del cuidado.

Instrumento $\mathrm{N}^{\circ}$ 2: Cuestionario elaborado por las autoras de este estudio sobre variables descriptivas personales y clínicas de usuarios que reciben cuidado de estudiantes de $5^{\circ}$ año de Enfermería: edad; sexo; situación de pareja; posición en la familia; escolaridad; motivo de atención en salud; experiencias previas de atención en salud.

Instrumento $\mathrm{N}^{\circ}$ 3: Cuestionario Caring Behaviors Inventory (CBI) Wolf, 1998, versión estudiantes, cuyo uso fue autorizado por Wolf.

Instrumento $\mathrm{N}^{\circ}$ 4: Cuestionario CBI. Wolf, 1998. Versión usuarios. Cuestionario Caring Behaviors Inventory (CBI), de la autora Zane Wolf, desarrollado en 1986, optimizado en 1998. Utilizado para evaluar el cuidado de enfermería. Actualmente establece 42 ítemes distribuidos en 5 subescalas o dimensiones, cada uno con un puntaje de 6 puntos en las 
siguientes categorías: $1=$ nunca; 2 = casi nunca; $3=$ ocasionalmente; $4=$ usualmente; $5=$ casi siempre y $6=$ siempre.

Las cinco subescalas o dimensiones en las que se distribuyen los 42 ítemes son las siguientes:

1. Deferencia respetuosa con el otro (respeto por el otro). Ítemes $1,2,3,4,7,8,9,10,11$, 15, 27 y 28. En total: 12.

2. Asegura la presencia humana (necesidades humanas y seguridad). Itemes 16,18 , $26,29,30,31,32,33,34,35,36$ y 37. En total: 12 .

3. Conectarse positivamente (disposición de ayuda optimista y constantemente de la enfermera con el otro). Ítemes 5, 6, 12, 13, 14, 17, 21, 23 y 25. En total: 9.

4. Conocimiento y destreza profesional (enfermera con conocimiento, pericia y expertas). Ítemes 19, 20, 22, 24 y 38. En total: 5.

5. Prestar atención a las experiencias de otros (aprecio del acumulo de experiencia única del otro). Ítemes 39, 40, 41 y 42. En total: 4.

\section{Control de Calidad del instrumento}

Fiabilidad del instrumento: Los instrumentos principales (CBI), enviados por la autora Zane Wolf, traducidos con anterioridad por tres académicos bilingües chilenos $y$, nuevamente, revisados por los investigadores, fueron sometidos a una retraducción antes de ser aplicados y posteriormente sometidos a análisis la objetividad de su medición y el resultado a un coeficiente de confiabilidad. En estudios anteriores, sus 42 ítemes agrupados en 5 subescalas presentan un Alfa de Cronbach en general superior a 0.8 , por lo que se consideró confiable para realizar la investigación.

El instrumento presentó un Alfa de Cronbach de 0,845 , por lo que es evidente la consistencia interna entre los ítemes que constituyen cada dimensión. Lo que es congruente con el Alfa de Cronbach de 0,827 resultante "versión estudiantes" y 0,795 para "versión usuarios" en el estudio (Cuadro 1).

Cuadro 1. Confiabilidad del Cuestionario Caring Behaviors Inventory (CBI).

\begin{tabular}{|c|c|c|c|}
\hline Dimensiones & ítemes & Total ítems & Alfa de Cronbach \\
\hline $\begin{array}{l}\text { 1. Deferencia respetuosa con el otro (respeto } \\
\text { por el otro). }\end{array}$ & $\begin{array}{l}1,2,3,4,7,8,9,10 \\
11,15,27,28\end{array}$ & 12 & $\begin{array}{l}0.8906 \\
0.826^{\star} \\
0,704^{\star *}\end{array}$ \\
\hline $\begin{array}{l}\text { 2. Asegura la presencia humana (necesidades } \\
\text { humanas y seguridad). }\end{array}$ & $\begin{array}{l}16,18,26,29,30 \\
31,32,33,34,35 \\
\quad 36,37\end{array}$ & 12 & $\begin{array}{l}0,9221 \\
0.877^{\star} \\
0.866^{* *}\end{array}$ \\
\hline $\begin{array}{l}\text { 3. Conectarse positivamente (disposición de } \\
\text { ayuda optimista y constantemente de la enfer- } \\
\text { mera con el otro). }\end{array}$ & $\begin{array}{l}5,6,12,13,14,17 \\
21,23,25\end{array}$ & 9 & $\begin{array}{l}0.8452 \\
0.816^{\star} \\
0.758^{\star *}\end{array}$ \\
\hline $\begin{array}{l}\text { 4. Conocimiento y destreza profesional (en- } \\
\text { fermera con conocimiento, pericia y expertas). }\end{array}$ & $19,20,22,24,38$ & 5 & $\begin{array}{l}0.8157 \\
0.808^{\star} \\
0.736^{\star *}\end{array}$ \\
\hline $\begin{array}{l}\text { 5. Prestar atención a las experiencias de otros } \\
\text { (aprecio del acumulo de experiencia única del } \\
\text { otro). }\end{array}$ & $39,40,41,42$ & 4 & $\begin{array}{l}0.8190 \\
0.808^{\star} \\
0,913^{\star *}\end{array}$ \\
\hline Alfa Escala Global & & & 0,845 \\
\hline
\end{tabular}

Alfa de Cronbach obtenido en el estudio ${ }^{*} \mathrm{CBI}$ estudiantes, ${ }^{* *} \mathrm{CBI}$ usuarios. 
Validez: Se realizó una búsqueda de instrumentos basados en la Teoría Transpersonal del Cuidado Humano de Watson. Se seleccionó el instrumento Caring Behaviors Inventory (Inventario de comportamientos de los que cuidan) utilizado para evaluar el cuidado de enfermería que cuenta con versión para pacientes, lo que permitió la medición de las variables dependientes en estudio. Se realizó prueba de validez facial debido a que este instrumento no había sido usado en Chile anteriormente, a través de prueba piloto en 10 estudiantes, e igual número de usuarios de la segunda rotación de internado de un hospital público de la región, para evaluar los ítemes y emitir un juicio sobre la comprensión de los mismos, y comprobar si eran entendidos en el contexto y lenguaje nacional. Luego fueron incorporados a la muestra. La validación facial o aparente, de contenido y de criterio fueron realizadas en estudios anteriores $(18,21)$.

Procesamiento de los datos: Los datos fueron ingresados a una planilla Excel y analizados con el software estadístico SAS (Statistical Analysis System). Las variables numéricas fueron representadas a través de medidas de tendencia central y de dispersión; las variables categóricas fueron representadas por su frecuencia y su porcentaje. Se verificó las propiedades distribucionales requeridas para estos análisis, la normalidad y la homogeneidad de varianza a través de los test de Shapiro-Wilk y Levene, respectivamente.

Aspectos éticos involucrados: Previo a la ejecución, el proyecto se sometió a evaluación del Comité de Ética de la Facultad de Medicina de la Universidad de Concepción y del Comité de Bioética y Buenas Prácticas Clínicas del Servicio de Salud Concepción, siendo aprobado por ambas entidades.

El consentimiento informado fue elaborado específicamente para estudiantes y usuarios, cumpliendo con los requerimien- tos éticos establecidos (22).

\section{RESULTADOS}

Los resultados descriptivos de las variables de esta investigación caracterizan a internas/os con edades entre 21 y 33 años, con predominancia del sexo femenino. Respecto a antecedentes, se observa que el mayor porcentaje ingresó a la carrera entre los años 2004 a 2007, permaneciendo entre 8 y 6 años cursando estudios antes de alcanzar el $5^{\circ}$ nivel correspondiente a internado. Se encuentran alumnos con mayor data de ingreso, principalmente en universidades privadas, donde el avance curricular se muestra de más lenta progresión. Los servicios clínicos donde desarrollan sus prácticas clínicas corresponden en su mayoría a servicios generales utilizados principalmente por estudiantes de universidades 2 y 3 , a diferencia de universidad 1 con utilización principal de otros campos clínicos en unidades de especialidad, lo que se relaciona con la subestimación de los comportamientos del cuidado de los estudiantes de parte de los usuarios.

Al caracterizar a usuarios receptores del cuidado, se observa un promedio de edad que alcanza los 54,6 años. Del total de 96 usuarios un $53,1 \%$ corresponde al sexo femenino. El nivel educacional de los encuestados muestra que la mayoría posee estudios de enseñanza media, seguida por un poco más de un tercio con estudios de enseñanza básica, una minoría de los usuarios posee estudios superiores $y$ tres usuarios sin estudios formales.

Cuando se determina la percepción de comportamientos del cuidado de internos y usuarios receptores del cuidado se encuentra que en la Dimensión 1 "Deferencia respetuosa con el otro", todos los internos presentan puntuación superior a 5, en escala 1-6. Sin embargo, cuando se consulta a los usuarios con respecto a comportamientos del cuidado de los internos de las universidades 2 y 3 , 
éstos fueron calificados con puntajes sobre 5 (5,31 DE 1,$00 ; 5,53$ DE 0,47 respectivamente) y los internos de la universidad 1 con 3,68 (DE 1,26).

Al determinar la percepción de comportamiento en la Dimensión 2 "Asegura la presencia Humana”, Nuevamente todos los internos refieren puntuación sobre 5 . Nuevamente los usuarios vuelven a calificar con mejor puntaje a los internos de las universidades 2 y 3 (5,27 DE 1,05; 5,41 DE 0,49, respectivamente) y los internos de la universidad 1 son calificados con 3,66 DE 1,62.

En la evaluación de la percepción de comportamiento en la Dimensión 3 "Conectarse positivamente", nuevamente todos los internos refieren puntuación sobre 5. Los usuarios calificaron con puntaje superior a 5 a los internos de las universidades 2 y 3 (5,39 DE 0,$83 ; 5,51 \mathrm{DE} 0,51$, respectivamente) y los in- ternos de la universidad 1 fueron calificados con 3,6 DE 1,18.

Al determinar la percepción de comportamiento en la Dimensión 4 "Conocimiento y destreza profesional”, nuevamente todos los internos se calificaron con puntuación sobre 5. Los usuarios nuevamente calificaron con mejor puntaje a los internos de las universidades 2 y 3 (5,71 DE 0,45; 5,53 DE 0,40, respectivamente) y los internos de la universidad 1 fueron calificados con 4,03 DE 1,23.

Al determinar la percepción de comportamiento en la Dimensión 5 "Prestar atención a las experiencias de otros", todos los internos se evaluaron con puntuación sobre 5 . Nuevamente los usuarios calificaron con mejor puntaje a los internos de las universidades 2 y $3(5,28$ DE 0,$99 ; 5,39$ DE 0,50 , respectivamente) y los internos de la universidad 1 fueron calificados con 3,69 DE 1,66 (Tabla 1).

Tabla 1. Percepción de comportamientos de cuidado de internos y usuarios receptores del cuidado por universidad, Concepción 2012.

\begin{tabular}{llccc}
\hline & Grupo & $\begin{array}{c}\text { Universidad 1 } \\
\text { Media D.E. }\end{array}$ & $\begin{array}{c}\text { Universidad 2 } \\
\text { Media D.E. }\end{array}$ & $\begin{array}{c}\text { Universidad 3 } \\
\text { Media D.E. }\end{array}$ \\
\hline Dimensión 1 & Internos & $5,35(0,43)$ & $5,53(0,39)$ & $5,68(0,33)$ \\
& Usuarios & $3,68(1,26)$ & $5,31(1,00)$ & $5,53(0,47)$ \\
\hline Dimensión 2 & Internos & $5,24(0,54)$ & $5,43(0,45)$ & $5,61(0,37)$ \\
& Usuarios & $3,66(1,62)$ & $5,27(1,05)$ & $5,41(0,49)$ \\
\hline Dimensión 3 & Internos & $5,27(0,37)$ & $5,21(0,49)$ & $5,46(0,41)$ \\
& Usuarios & $3,6(1,18)$ & $5,39(0,83)$ & $5,51(0,51)$ \\
\hline Dimensión 4 & Internos & $5,05(0,73)$ & $5,08(0,47)$ & $5,38(0,40)$ \\
& Usuarios & $4,03(1,23)$ & $5,71(0,45)$ & $5,53(0,40)$ \\
\hline Dimensión 5 & Internos & $5,31(0,66)$ & $5,54(0,37)$ & $5,75(0,34)$ \\
& Usuarios & $3,69(1,66)$ & $5,28(0,99)$ & $5,39(0,50)$ \\
\hline
\end{tabular}

En relación a los comportamientos del cuidado brindado, referidos por internos, muestra para las dimensiones 1, 2 y 5 una sobreestimación de percepción de los estudiantes acerca del cuidado brindado, por sobre la estimación percibida por los usuarios.

Se observa en la dimensión 4, "conocimiento y destreza profesional", que los estudiantes de las universidades 2 y 3 subestiman sus comportamientos de cuidado respecto 
a lo estimado por los usuarios (Tabla1). El estudiante tiene claro que aún está en periodo de aprendizaje, y ejerce autocrítica sobre comportamientos derivados del conocimiento científico y técnico, a su vez, el usuario sobreestima éstos, por asociarlos a las otras dimensiones del cuidado.

\section{DISCUSIÓN Y CONCLUSIÓN}

El instrumento Inventario de Comportamientos de los que Cuidan "versión estudiantes" y "versión usuarios" de Zane Wolf, en español, presenta validez facial o aparente, al aplicarlo a los estudiantes y usuarios, sus ítemes fueron claramente comprendidos. De acuerdo con los resultados de validez y confiabilidad, se muestra que el instrumento es apto para ser utilizado en el contexto chileno. Dando pautas para iniciar nuevos campos de investigación con el fin de evaluar y orientar los procesos docentes, la práctica de enfermería y una herramienta para la acreditación de las instituciones sanitarias y educacionales en la relación docente-asistencial. Además, esta validación inicial entrega evidencia acerca de la importancia de obtener resultados de más de un actor del ejercicio del cuidado, como medio útil y efectivo de validación de instrumentos utilizados.

Algunos de los resultados de este estudio muestran que los comportamientos del cuidado difieren según los modelos que guíen la formación práctica y las oportunidades de ejercicio de conocimientos y destreza profesional. Es así como los estudiantes de la universidad 1 fueron evaluados por los receptores del cuidado con menor puntaje que los de las universidades 2 y 3 , que disponen de mayores oportunidades de modelaje y prácticas clínicas. La evidencia disponible respecto a lo anteriormente mencionado confirma esto y añade que el modelamiento incluso podría ocasionar que se reduzcan estos comportamientos en cursos superiores $(15,17)$.
Ramírez y Parra (17) determinaron que dentro de los comportamientos del cuidado existía una correlación positiva en categorías de: ser accesible, monitorea y mantiene relación de confianza; lo que estaría de acuerdo con los postulados para la práctica del internado, donde la formación humanística y ética son aspectos esenciales mostrados a través de las relaciones humanas de los actores del cuidado (23). Mlinar (16) al respecto considera la importancia de los comportamientos del cuidado de las enfermeras asistenciales y las docentes, en la preparación de los estudiantes para el desarrollo de las prácticas clínicas.

Otro aspecto importante a considerar de este estudio es que la puntuación menor que recibieron los estudiantes de la universidad 1 podría tener relación con que su desempeño de internado se realizó en servicios especializados, en donde con frecuencia el mayor énfasis está vinculado a la atención, demostrando conocimiento y destreza. Este es un aspecto que valdría la pena averiguar posteriormente en otros estudios.

Las diferencias de percepción de los usuarios y de los internos son esperables. Según la propia experiencia se moldea la conducta en los internos y esto hace que valoren ( $\mathrm{y}$ a veces sobrevaloren) de determinada manera el cuidado que realizan, sin embargo, los usuarios también tienen sus propias percepciones y son éstas las que finalmente deberían ser consideradas para reorientar y retroalimentar la formación de futuros enfermeros, como también lo señalan Murphy et al. (18). Leininger establece diferencias entre cuidados genéricos, cuidados profesionales y cuidados profesionales de enfermería (23). El estudiante, al tener claro que se encuentra aún en período de aprendizaje, es autocrítico especialmente en los comportamientos derivados de ejercicio de conocimientos y destreza profesional, a su vez, el usuario estaría en mejor condición para evaluar los otros aspectos del cuidado señalados en las dimensiones 1,2 y 4 . 
Resulta un desafío en sí mismo el evaluar aspectos tan cualitativos del cuidado, como los que emergen de la visión de Watson, en una investigación de abordaje cuantitativo. El instrumento de Wolf permitió realizar esto, por lo que es recomendable continuar investigando, considerando la incorporación del binomio enfermera/estudiante de enfermería y receptor del cuidado.

\section{REFERENCIAS}

1. Comisión Investigadora sobre el Funcionamiento de la Educación Superior. Informe Ministerio de Educación División Educación Superior [Internet]. Chile: Gobierno de Chile, Ministerio de Educación; oct 2011 [citado 05 septiembre 2011]. 33 p. Disponible en: http://www. elmostrador.cl/media/2012/12/Informe-Comision-Lucro-parte-I.pdf

2. Pavez K. Enfermería iguala salarios de ingenieros al año de egreso y se consolida entre las más cotizadas del mercado. La Tercera. 23 de agosto 2009: Educación. p. 35.

3. Urbina O, Pérez A, Delgado JE, Martín X. El modelo de actuación de Enfermería y su valor humanista. Educ Med Super [Internet]. 2003 [citado 05 septiembre 2011]; 17(3):0-0 Disponible en: arttext\&pid $=$ S0864-21412003000300002\&ln$\mathrm{g}=\mathrm{es}$

4. Ceballos P. Desde los ámbitos de enfermería, analizando el cuidado humanizado. Cienc. enferm. 2010; XVI (1): 31-35.

5. Pinto N. Nuevos avances conceptuales del Grupo de Cuidado. En: Grupo de Cuidado. Cuidado y Práctica de Enfermería. Bogotá: Universidad Nacional de Colombia; 2000. p. 4-13.

6. Vieira de Almeida D, Corrêa E. Teaching humanization in undergraduate nursing course subjects. Invest Educ Enferm. 2013; 31(1): 44-53.
7. Medina J. La Pedagogía del Cuidado. Saberes y prácticas en la formación universitaria en enfermería. Barcelona: Editorial Laertes; 1999. 307 p.

8. Watson J. Ponencia del Primer Congreso Internacional de Enfermería. Facultad de Enfermería y Nutriología. México: Universidad Autónoma de Chihuahua; 2007.

9. Díaz M, Castro DM, Cuevas BL. Valores profesionales de enfermería: Una mirada hacia la formación en la Educación Superior. Humanidades Médicas [Internet]. 2012 [citado 11 jul 2013]; 12(2): 289-99. Disponible en: http://scielo.sld.cu/pdf/ hmc/v12n2/hmc11212.pdf

10. Organización Panamericana de la Salud, Organización Mundial de la Salud, Área de Fortalecimiento de Sistemas de Salud, Unidad de Recursos Humanos para la Salud. Orientaciones para la Educación Inicial de Enfermería en las Américas: hacia el 2020 (Documento en Proceso de Elaboración) [Internet]. Washington: OPS, OMS; Sep 2007 [citado 30 ago 2012]. 37 p. Disponible en: http://www.eean.ufrj. br/aladefe/orientaciones.pdf

11. Ley $\mathrm{N}^{\circ}$ 20.584. Regula los Derechos y Deberes que tienen las personas en relación con acciones vinculadas a su atención en Salud. D.O. 26 julio 2006. [citado 11 jul 2013]. Disponible en: http:// web.minsal.cl/portal/url/item/cbb3d986537436a2e040010165015aa4.pdf

12. Gómez OJ, Daza LA, Zambrano VM. Percepción de cuidado en estudiantes de enfermería: caracterización e impacto para la formación y la visión del ejercicio profesional. av. enferm. 2008; XXVI(1): 8596.

13. Marriner A, Raile M. Modelos y Teorías en Enfermería. 6a ed. Madrid: Elsevier Mosby; 2007.828 p.

14. Poblete M, Valenzuela S, Merino JM. Validación de dos escalas utilizadas en la medición del cuidado transpersonal basadas en la Teoría de Jean Watson. Aquichan. 2012; 12(1): 8-21. 
15. Urra E, Jana A, García M. Algunos aspectos esenciales del pensamiento de Jean Watson y su teoría de cuidados transpersonales. Cienc. enferm. 2011; XVII (3): 11-22.

16. Mlinar S. First- and third-year student nurses' perceptions of caring behaviours. Nurs Ethics. 2010; 17(4): 491-500.

17. Ramírez CA, Parra M. Percepción de los comportamientos del cuidado de enfermería en la unidad de cuidados intensivos. av.enferm. 2011; XXIX (1): 97-108.

18. Murphy F, Jones S, Edwards M, James J, Mayer A. The impact of nurse education on the caring behaviours of nursing students. Nurse Educ Today. 2009; 29(2):254-64.

19. Camargo IL, Caro CV. Comportamientos de cuidado del anciano y de los profesionales de enfermería: evidencias para la cualificación profesional. Provincia de Coclé, Panamá. av.enferm. 2009; XXVII(1): 48-59.

20. Rivera LN; Triana A. Percepción de comportamientos de cuidado humanizado de enfermería en la Clínica del Country. av. enferm. 2007; XXV(1):56-68.

21. Hernández R, Fernández C, Baptista P. Metodología de la investigación. 2da ed. México: Mc Graw-Hill; 1998. 501 p.

22. Emanuel E. ¿Qué hace que la investigación clínica sea ética? Siete requisitos éticos. En: Pellegrini A, Macklin R, editores. Investigación en Sujetos Humanos: Experiencia Internacional. Santiago, Chile: Programa Regional de Bioética OPS/ OMS; 1999. p 33-46.

23. Leininger M. Teoría de los cuidados culturales. En: Marriner A, Raile M. Modelos y Teorías de Enfermería. 6a ed. Philadelphia: Mosby; 2007.p. 472-98. 